\title{
IGF-II/mannose 6-phosphate receptor activation induces metalloproteinase-9 matrix activity and increases plasminogen activator expression in $\mathrm{H} 9 \mathrm{c} 2$ cardiomyoblast cells
}

\author{
Mu-Hsin Chang ${ }^{1,2}$, Wei-Wen Kuo ${ }^{3}$, Ray-Jade Chen ${ }^{4}$, Ming-Chin Lu ${ }^{5}$, Fuu-Jen Tsai ${ }^{6}$, \\ Wu-Hsien Kuo ${ }^{7}$, Ling-Yun $\mathrm{Chen}^{8}$, Wen-Jun $\mathrm{Wu}^{2, \star}$, Chih-Yang Huang ${ }^{9,10,11, *}$ \\ and Chun-Hsien $\mathrm{Chu}^{8,10, *}$ \\ ${ }^{1}$ Division of Cardiology, Armed Force Taichung General Hospital, Taichung, Taiwan, ROC \\ ${ }^{2}$ Institute of Medical and Molecular Toxicology, Chung Shan Medical University, Taichung, Taiwan, ROC \\ ${ }^{3}$ Department of Biological Science and Technology, China Medical University, Taichung 404, Taiwan, ROC \\ ${ }^{4}$ Trauma and Emergency Center, China Medical University Hospital, Taichung 404, Taiwan, ROC \\ ${ }^{5}$ Post-Baccalaureate School of Chinese Medicine, China Medical University, Taichung 404, Taiwan, ROC \\ ${ }^{6}$ Department of Pediatrics, Medical Research and Medical Genetics, China Medical University, Taichung, Taiwan, ROC \\ ${ }^{7}$ Division of Gastroenterology, Department of Internal Medicine, Armed Force, Taichung General Hospital, Taichung, Taiwan, ROC \\ ${ }^{8}$ Institute of Biochemistry and Biotechnology, Chung Shan Medical University, Taichung 402, Taiwan, ROC \\ ${ }^{9}$ Graduate Institute of Chinese Medical Science and ${ }^{10}$ Institute of Basic Medical Science, China Medical University, Taichung 404, Taiwan, ROC \\ ${ }^{11}$ Department of Health and Nutrition Biotechnology, Asia University, Taichung 413, Taiwan, ROC
}

(Correspondence should be addressed to C-H Chu who is now at Graduate Institute of Basic Medical Science, China Medical University, No. 91, Hsueh-Shih Road, Taichung 404, Taiwan, ROC; Email: s210001@ smail.csmu.edu.tw)

*W-J Wu, C-Y Huang and C-H Chu contributed equally to this work

\begin{abstract}
The IGF-II/mannose 6-phosphate receptor (IGF2R) function in extracellular matrix (ECM) remodeling is known to occur as a result of transforming growth factor- $\beta$ (TGF- $\beta$ ) activation and plasmin in the proteolytic cleavage level caused by the interaction between latent TGF- $\beta$ and urokinase plasminogen activator receptor (UPAR) respectively. In one of our previous studies, we found IGF-II and IGF2R dose-dependently correlated with the progression of pathological hypertrophy remodeling following complete abdominal aorta ligation. However, how this IGF2R signaling pathway responds specifically to IGF-II and regulates the myocardial ECM remodeling process is unclear. We found that IGF2R was aberrantly expressed in myocardial infarction scars. The matrix metalloproteinase-9 (MMP-9) zymographic activity was elevated in H9c2 cardiomyoblast cells treated with IGF-II, but not IGF-I. Treatment with Leu27IGF-II, an IGF2R specifically binding IGF-II analog, resulted in significant time-dependent increases in the MMP-9, tissue-type plasminogen activator (tPA), and urokinase plasminogen activator (UPA); and a reduction in the tissue inhibitor of matrix metalloproteinases-2 (TIMP-2) protein expression. Furthermore, IGF2R expression inhibition by siRNA blocked the IGF-II-induced MMP-9 activity. We hypothesize that after IGF-II is bound with IGF2R, the resulting signal disrupts the balance in the MMP-9/TIMP-2 expression level and increases plasminogen activator (PAs) expression involved in the development of myocardial remodeling. If so, IGF2R signaling inhibition may have potential use in the development of therapies preventing heart fibrosis progression.
\end{abstract}

Journal of Molecular Endocrinology (2008) 41, 65-74

\section{Introduction}

When responding to pathological stresses, e.g., hypertension and myocardial infarction, the heart undergoes a myocardial extracellular matrix (ECM) remodeling that brings about decreased microvasculature and increased ECM deposition, which may in turn contribute to heart failure development (Berk et al. 2007, Gallagher et al. 2007). The ECM degrading enzymes such as plasminogen activation (PA) system and the matrix metalloproteinase (MMP) system (Cleutjens \& Creemers 2002, Spinale
2002) are increased in animals and patients with systolic heart failure (Spinale et al. 2000, Gaertner et al. 2005). The activities of these two systems are tightly modulated by their physiological inhibitors: the plasminogen activator inhibitors (PAIs) and tissue inhibitor of matrix metalloproteinases (TIMPs; Li et al. 2000a, Fay 2004). According to two previous gene knockout animal studies, the decreased expression of either urokinase PA (uPA) or MMP-9 in the heart can inhibit cardiac rupture after myocardial infarction, indicating that increased PAs or MMP-9 expression may lead to cardiac rupture 
(Heymans et al. 1999, Ducharme et al. 2000). Insulin-like growth factor 2 receptor (IGF2R) has been reported to directly complex with latent transforming growth factor- $\beta$ (TGF- $\beta$ ), plasminogen, and uPAR (Odekon et al. 1994, Godar et al. 1999, Ghosh et al. 2003, Leask \& Abraham $2004)$. It contributes to the activation of TGF- $\beta$ and the conversion of plasminogen into plasmin. TGF- $\beta$, a powerful inducer of matrix gene expression in many cell types, plays an integral role in fibrogenesis (Leask \& Abraham 2004), indicating that IGF2R may play an important role in myocardial remodeling fibrinolysis.

IGF2R, a $300-\mathrm{kDa}$ type I transmembrane glycoprotein, triggers various cellular functions by interacting with several distinct classes of ligands, including IGF-II and other proteins containing Man-6-P on carbohydrate side chains (Jones \& Clemmons 1995). In a study of transgenic mice, the lack of an IGF-II/M6P receptor was associated with over proliferation of myocardial cells in ventricular hyperplasia (Lau et al. 1994). Furthermore, IGF2R protein ribozyme disruption protects cardiac myocytes against hypoxia- and TNF-induced apoptosis (Chen et al. 2004), suggesting that the IGF2R expression level in the heart has a vital role in the regulation of cardiac development, growth, and survival either in the embryo or in the adult. The classical function of IGF2R in the control of IGF-II concentrations through internalization and lysosomal degradation may suppress mitogenesis by reducing the availability of IGF-II, which binds to the IGF-I receptor (Jones \& Clemmons 1995). Recently, it has been found that there is a putative G-protein binding site within the cytoplasmic domain of IGF2R and that IGF-II binding with IGF2R activates a G-protein sensitivedependent pathway that contributes to a variety of physiological functions (Nishimoto et al. 1987, McKinnon et al. 2001, Hawkes et al. 2006). In our previous study, the upregulation of IGF-II and IGF2R genes were detected in rats made hypertensive by abdominal aorta ligation and $\mathrm{H} 9 \mathrm{c} 2$ cardiomyoblast cells treated with ANGII (Lee et al. 2006). Based on these findings, we hypothesized that the binding of IGF-II to IGF2R might regulate myocardial remodeling through activating intracellular signaling.

We investigated whether IGF-II binding to IGF2R might be involved in myocardial remodeling through the regulation of ECM degrading enzymes. We found that IGF2R was aberrantly expressed in myocardial scar tissue. In our study on $\mathrm{H} 9 \mathrm{c} 2$ cardiomyoblast cell cultures we used gelatin zymography to measure MMP-9 and MMP-2 activity in cells treated with IGF-I, IGF-II, and Leu27IGF-II, an IGF2R specifically binding IGF-II analog (Beukers et al. 1991). Treatment with both IGF-II and Leu27IGF-II, but not IGF-I, induced an increase in MMP-9 activity. Western blot revealed that treatment with Leu27IGF-II not only increased MMP-9, tPA, and uPA protein expression but also reduced
TIMP-2 protein expression in $\mathrm{H} 9 \mathrm{c} 2$ cardiomyoblast cells. The inhibition of IGF2R expression by siRNA blocked the IGF-II-induced MMP-9 activity.

Taken together, our findings suggested that the IGF2R signaling pathway may contribute to the progression of myocardial remodeling by disrupting the balance in MMP-9/TIMP-2 expression level and increasing PAs expression. Hopefully, the new insights provided by this study may be used to prevent chronic heart disease associated with fibrosis.

\section{Materials and methods}

\section{Immunohistochemical analysis}

Human cardiovascular tissue array (Provitro, Berlin, Germany) was immunostained with an anti-IGF2R antibody (SantaCruz Biotechnology, SantaCruz, CA, USA) using an Ultra Vision LP Detection System (Vector Laboratories, Burlingame, CA, USA) according to the manufacturer's instructions. The human cardiovascular tissue array was dried at $58^{\circ} \mathrm{C}$ overnight following deparaffinization in xylene and hydrated using a graded series of ethanol. Endogenous peroxidase activity was blocked using hydrogen peroxide blocking buffer for $13 \mathrm{~min}$. After rinsing in water for $15 \mathrm{~min}$, the microarray slide was microwave treated in citrate buffer for $15 \mathrm{~min}$, cooled at room temperature (RT) for $30 \mathrm{~min}$ and blocked with an ultra $\mathrm{V}$ blocking buffer for $5 \mathrm{~min}$. The primary antibody directed against the peptides 1030-1209 in the rat IGF2R (1:100) was incubated for $30 \mathrm{~min}$. The slide was incubated with primary antibody enhancing buffer at RT for $20 \mathrm{~min}$. HRP Polymer was added and incubated at RT for $20 \mathrm{~min}$. The IGF2R antibody was located using a universal secondary antibody formulation conjugated to an enzyme-labeled HRP Polymer. After staining with an appropriate substrate/chromogen for $5 \mathrm{~min}$, the slide was counterstained with Harris hematoxylin, dehydrated through a graded series of ethanol to xylene washes, and coverslipped with permanent mounting media (Sigma Chemical). The polymer complex was then detected using microscopy (magnification $200 \times$ ).

\section{Cell culture}

H9c2 cardiomyoblast cells were obtained from American Type Culture Collection (ATCC) and cultured in Dulbecco's modified essential medium supplemented with $10 \%$ fetal bovine serum, $2 \mathrm{mM}$ glutamine, $100 \mathrm{U} / \mathrm{ml}$ penicillin, $100 \mu \mathrm{g} / \mathrm{ml}$ streptomycin, and $1 \mathrm{mM}$ pyruvate in humidified air $\left(5 \% \mathrm{CO}_{2}\right)$ at $37^{\circ} \mathrm{C}$. $\mathrm{H} 9 \mathrm{c} 2$ cells were cultured in serum-free medium for $12 \mathrm{~h}$ and then treated with or without IGF-I $\left(10^{-8} \mathrm{M}\right.$; Sigma Chemical), IGF-II $\left(10^{-8} \mathrm{M}\right.$; Sigma Chemical) or Leu27IGF-II $\left(10^{-8} \mathrm{M}\right.$; GroPep, Adelaide, Australia). 
After further incubation for $12 \mathrm{~h}$ or $24 \mathrm{~h}$, the cells were harvested and extracted for analysis.

\section{Protein extraction and western blot analysis}

Cultured H9c2 cells were scraped and washed once with PBS. The cell suspension was then centrifuged and the cell pellets lysed for $30 \mathrm{~min}$ in lysis buffer $(50 \mathrm{mM}$ Tris, $\mathrm{pH} 7 \cdot 5,0.5 \mathrm{M} \mathrm{NaCl}, 1.0 \mathrm{mM}$ EDTA, pH 7.5, $10 \%$ glycerol, $1 \mathrm{mM}$ basal medium Eagle, 1\% Igepal-630, and proteinase inhibitor cocktail tablet (Roche)) and centrifuged at $12000 \mathrm{~g}$ for $10 \mathrm{~min}$. The supernatants were removed and placed in new Eppendorf tubes for western blot analysis. Proteins from the H9c2 cell line were separated in $12 \%$ gradient SDS-PAGE and transferred onto nitrocellulose membranes. Nonspecific protein binding was blocked in blocking buffer at RT for $1 \mathrm{~h}(5 \%$ milk, $20 \mathrm{mM}$ Tris-HCl, $\mathrm{pH} 7 \cdot 6,150 \mathrm{mM}$ $\mathrm{NaCl}$, and $0 \cdot 1 \%$ Tween 20 ). The membranes were blotted with specific uPA (SantaCruz Biotechnology), Akt (SantaCruz Biotechnology), p-Akt (SantaCruz Biotechnology), tPA (SantaCruz Biotechnology), PAI-1 (SantaCruz Biotechnology), TGF- $\beta$ (SantaCruz Biotechnology), MMP-9 (SantaCruz Biotechnology), MMP-2 (SantaCruz Biotechnology), TIMP-1 (SantaCruz Biotechnology), TIMP-2 (SantaCruz Biotechnology), and $\alpha$-tubulin (SantaCruz Biotechnology) antibodies and incubated in $4{ }^{\circ} \mathrm{C}$ blocking buffer overnight. Densitometric analysis of immunoblots was performed using the AlphaImager 2200 digital imaging system (Digital Imaging System, CA, USA). Experiments were performed in triplicate.

\section{Gelatin zymography}

Cell mediums collected from H9c2 cardiomyoblast cells after treatment were diluted in non-reducing $2 \%(\mathrm{w} / \mathrm{v})$ SDS sample buffer and electrophoresed on $10 \%$ polyacrylamide SDS gels containing $0 \cdot 1 \%(\mathrm{w} / \mathrm{v})$ gelatin (Sigma Chemical). After electrophoresis, gels were washed at RT for $2 \times 30 \mathrm{~min}$ in $2.5 \%(\mathrm{v} / \mathrm{v})$ Triton X-100 to remove SDS and incubated at $37^{\circ} \mathrm{C}$ for $24 \mathrm{~h}$ in $50 \mathrm{mM}$ Tris- $\mathrm{HCl}$ buffer, $\mathrm{pH} 7 \cdot 5$, containing $200 \mathrm{mM} \mathrm{NaCl}$, and $5 \mathrm{mM} \mathrm{CaCl}$. After incubation, gels were stained for 30 min with $0 \cdot 1 \%$ (w/v) G-250 Coomassie Blue in $45 \%$ (v/v) methanol, $10 \%(\mathrm{v} / \mathrm{v})$ acetic acid glacial, and destained in the same solution without dye. All experiments were performed in triplicate.

\section{Total RNA extraction and RT-PCR}

Total RNA was extracted using the Ultraspec RNA isolation system (Biotecx Laboratories, Houston, TX, USA) according to directions supplied by the manufacturer. The RNA precipitate was washed twice using gentle vortexing with $70 \%$ ethanol, collected by centrifugation at $12000 \mathrm{~g}$, dried under vacuum for 5-10 min, dissolved in 50-100 $\mu \mathrm{l}$ diethylpyrocarbonatetreated water, and incubated for $10-15 \mathrm{~min}$ at $55-60^{\circ} \mathrm{C}$. cDNA was prepared in a buffer containing $50 \mathrm{mM}$ Tris$\mathrm{HCl}, \mathrm{pH} 8 \cdot 5,30 \mathrm{mM} \mathrm{KCl}, 8 \mathrm{mM} \mathrm{MgCl}, 1 \mathrm{mM}$ dithiothreitol, $0.25 \mathrm{mM}$ each dCTP, dGTP, dTTP, and dATP, $20 \mathrm{U}$ recombinant ribonuclease inhibitor, $1 \mathrm{pg}$ random hexamers, 5 pg total RNA, and $40 \mathrm{U}$ avian myeloblastosis virus reverse transcriptase in a volume of $20 \mathrm{pl}$. This mixture was incubated for $10 \mathrm{~min}$ at RT followed by $1 \mathrm{~h}$ at $42{ }^{\circ} \mathrm{C}$ to initiate cDNA synthesis. This mixture was then used for amplification of specific cDNAs by PCR. The PCR buffer contained $50 \mathrm{mM}$ KCI, $10 \mathrm{mM}$ Tris-HC1, pH 8.3, at $20{ }^{\circ} \mathrm{C}, 0.2 \mathrm{mM}$ each dCTP, dGTP, dTMP, and dATP, $0.5 \mathrm{pM}$ oligonucleotide PCR primers, $2.5 \mathrm{U}$ Taq polymerase, and various $\mathrm{MgCl}$ concentrations in a final volume of $100 \mathrm{pl}$. Following the hot start $(5 \mathrm{~min}$ at $95^{\circ} \mathrm{C}, 80{ }^{\circ} \mathrm{C}$ hold $)$, the samples were subjected to 35 cycles of $45 \mathrm{~s}$ at $95{ }^{\circ} \mathrm{C}, 2 \mathrm{~min}$ at $52^{\circ} \mathrm{C}$, and $45 \mathrm{~s}$ at $72^{\circ} \mathrm{C}$. For the MMP-9, TIMP-2, uPA, tPA and GAPDH primers, the primer annealing temperature was $56^{\circ} \mathrm{C}$. This was followed by a final extension step at $72^{\circ} \mathrm{C}$ for $10 \mathrm{~min}$. All RNA samples used were demonstrated to have intact $18 \mathrm{~S}$ and 28S RNA bands on ethidium bromide-strained formaldehyde-agarose gels. Primers were as follows: rat MMP-9 forward primer CAGTTTGGTGTCGCGGAGCA, reverse primer AGGCCATGGGAGGTGCAGTG; rat TIMP-2 forward primer GGGTCTCGCTGGACGTTGGA, reverse primer AACTCCTGCTTCGGGGGTGC; rat uPA forward primer ACTCATCCCCACGCTGACCG, reverse primer AGTGGCCCTTACCCCACCCA; rat tPA forward primer ACACAGCGTGGAGGGCCAAC, reverse primer AGGATGCCTCATGCTTGCCG; rat GAPDH forward primer TCCCTCAAGATTGTCAGCAA, reverse primer AGATCCACAACGGATACATT (MDBio, Taipei, Taiwan).

\section{siRNA and transfection}

Double-stranded siRNA sequences targeting IGF2R mRNAs were obtained from Dharmacon. A non-specific duplex (5'-CAGUGGAGAUCAACGUGCAAGUU-3'; Dharmacon) was used as a control. NRVM were plated in 100-mm well plates in DMEM without fetal bovine serum and transfected with double-stranded siRNA using the DharmaFECT Duo Transfection Reagent (Dharmacon) according to the manufacturer's instructions. To assess gene silencing, the IGF2R protein level was detected by immunoblotting.

\section{Densitometry and statistical analysis}

The relative protein intensities and MMP-2/9 activity were analyzed using the Digital Sciences 1D program from Kodak Scientific Imaging Systems (New Haven, 
CT, USA). All the results were expressed as means \pm s.D. or the means and coefficient of variation of three to five separate experiments as indicated. The transfection experiments were performed in triplicate. Standard curves were run and the data that were obtained fell within the linear range of the curve. In addition, all values were normalized to their respective lane load controls. The densitometric analysis of immunoblots and gelatin zymography in bar Fig. 5b were analyzed using one-way ANOVA with preplanned contrast comparisons against the control group (serum free) or against the IGF-II group. Results in Figs 2, 3b, d, 4b, d and 5 a were analyzed by unpaired Student's $t$-test. In all cases, $P<0.05$ was considered significant.

\section{Results}

\section{Expression analysis of IGF2R in the myocardial scar tissues}

To examine the IGF2R protein expression level during myocardial remodeling, we performed immunochemistry analysis of human cardiovascular tissue array containing ten normal heart and ten myocardial scar tissues. Representative images demonstrating positive or negative myocardial scar staining compared with normal human heart tissue are shown in Fig. 1. A total of nine $(45 \%)$ showed positive staining for IGF2R. Thus, $11(55 \%)$ could be categorized as absent or minimal expression for IGF2R. Out of the myocardial scar samples, five showed strong expression of IGF2R $(50 \%)$ and four showed moderate expression (40\%). Only one out of the ten myocardial infarction samples appeared to have no more staining than normal heart tissue. None of the ten normal tissue samples on the slide showed any IGF2R overexpression. Overall, then, nine out of ten myocardial scars $(90 \%)$ examined by immunohistochemistry showed significant overexpression of IGF2R. Taken together, our finding showed that the IGF2R was aberrantly expressed in myocardial infarction scars.

\section{IGF-II induction of endogenous MMP-9 release from H9c2 cardiomyoblast cells}

We investigated whether treatment with IGF-II would directly influence MMP-9 zymographic activity in H9c2 cardiomyoblast cells, and compared its effect with that of IGF-I. Representative gelatin zymography assays of culture mediums taken from H9c2 cardiomyoblast cells treated respectively with IGF-I and IGF-II. Gelatin zymography revealed, when compared with untreated controls, that there was a sixfold increase in MMP-9 activity, but not MMP-2, in cells treated with IGF-II (Fig. 2a and b). However, in cells treated with IGF-I, there was no difference between the MMP-9 and MMP-2 activities (Fig. 2a and b). Increased MMP-9 was detected only in the cells treated with IGF-II, indicating that IGF2R plays a crucial role in MMP-9 activity induction. Furthermore, using Leu27IGF-II to excludes other effects derived from insulin and IGF-I receptor. We attempted to clarify whether the IGF-II-induced MMP-9 activity is mediated through IGF2R. Western blots revealed treatment with IGF-I and IGF-II, but not Leu27IGF-II, increased AKT phosphorylation at 30 min (Fig. 2c), suggesting that Leu27IGF-II did not activate IGF-I receptor downstream effectors. Although the Leu27IGF-II function in MMP-9 activity induction within $24 \mathrm{~h}$ is similar to that of IGF-II, cells treated only with Leu27IGF-II for $12 \mathrm{~h}$ showed significantly elevated

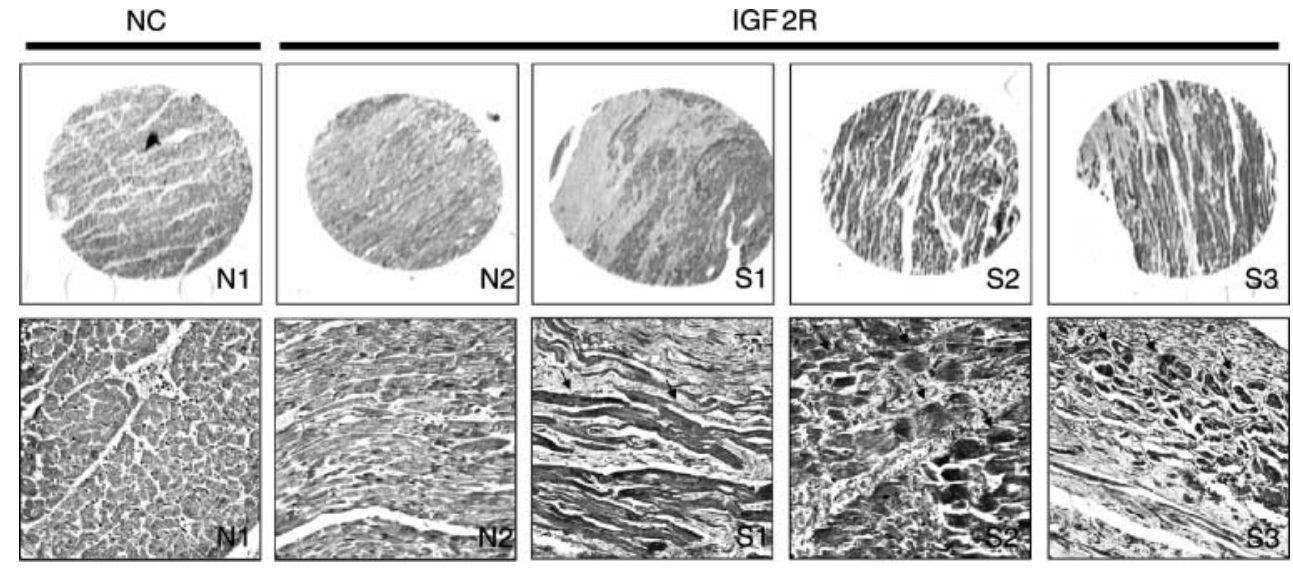

Figure 1 Detection of IGF2R expression in human cardiovascular tissue array by immunohistochemistry (IHC). The panel labeled N1 stains with anti-IgG antibody in a normal heart section, indicated as a negative control (NC). The panel labeled N2 shows the IGF2R antibody staining weakly in a normal heart section. Panels S1-S3 shows myocardial infarction staining strongly with the anti-IGF2R antibody, which is indicated by arrows. Magnification: $\sim \times 50$ (upper panel); $\times 200$ (bottom panel). 
(a)
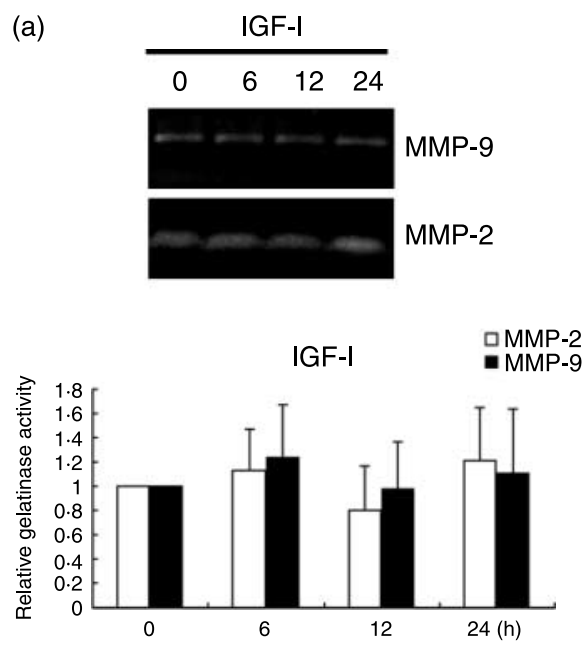

(c)
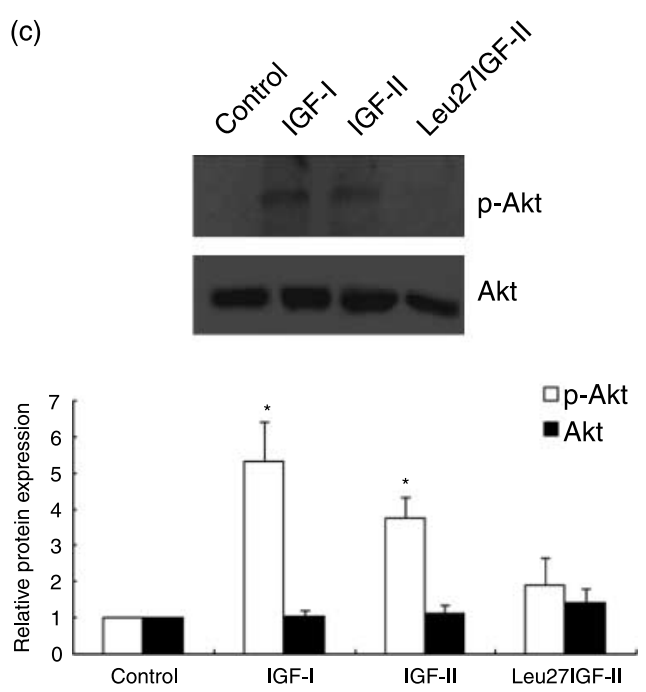

(b)
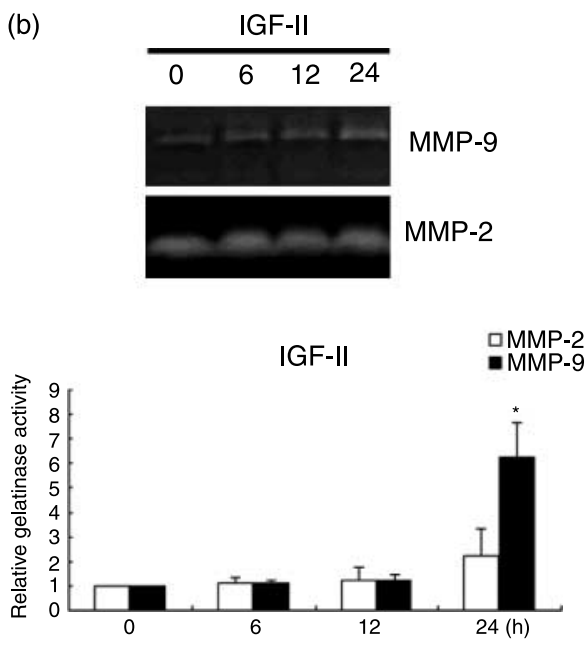

(d)
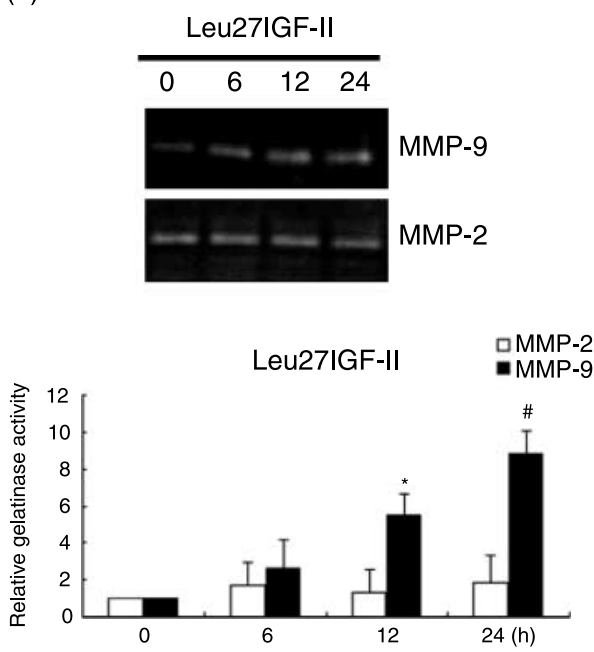

Figure 2 IGF-I and IGF-II differentially regulates endogenous MMP-9 release from the H9c2 cardiomyoblast cell. (a) Western blot analysis of H9c2 cardiomyoblast cell treated with IGF-I $\left(10^{-8} \mathrm{M}\right)$ or IGF-II $\left(10^{-8} \mathrm{M}\right)$ or Leu27IGF-II $\left(10^{-8} \mathrm{M}\right)$ respectively in 30 min using anti-phosphoAkt antibody, anti-Akt antibody, and anti- $\alpha$-tubulin antibody. Phospho-Akt protein levels increased treatment with IGF-I or IGF-II, but not with treatment with Leu27IGF-II. These bolts were quantified by densitometry. $\alpha$-Tubulin served as a loading control. Data are presented as means \pm S.E.M. of three independent experiments for each data points. Bars indicate averages, ${ }^{*} P<0.05$ was based on comparisons with untreated control. (b-d) Cell mediums collected from H9c2 cardiomyoblast cell treated with IGF-I (10 ${ }^{-8} \mathrm{M}$ ) or IGF-II (10 ${ }^{-8}$ M) or Leu27IGF-II $\left(10^{-8} \mathrm{M}\right)$ respectively for indicated time to detect the MMP-2/-9 activity by gelatin zymography. The results showed that IGF-I, IGF-II, and Leu27IGF-II have no effect on MMP-2 activity. The MMP-9 activity was significantly increased both in the treatment with Leu27IGF-II for $12 \mathrm{~h}$ and IGF-II for $24 \mathrm{~h}$ compared with untreated controls, whereas no effect on MMP-9 activity in IGF-I treatment. Data are quantified by densitometry and expressed as fold change of untreated control. Shown are mean results \pm S.E.M. of three independent experiments performed in duplicate. Statistical significance: ${ }^{\star} P<0.05 ;{ }^{\#} P<0.01$, IGF-I or IGF-II or Leu27IGF-II treated versus untreated controls.

MMP-9 activity (Fig. 2d). Taken together, our finding indicated that IGF-II-induced MMP-9 activity occurs specifically through activating IGF2R signaling and that may be involved in myocardial remodeling.

\section{Leu27IGF-II modulation of the MMP-9/TIMP-2 and PAs expression}

To investigate whether the IGF2R signaling may regulate ECM degrading enzyme systems that contribute to myocardial fibrosis, we further used Leu27IGF-II to specifically activate IGF2R-deriving signaling and detection of MMPs, TIMPs, and PAs in the level of mRNA and protein in $\mathrm{H} 9 \mathrm{c} 2$ cardiomyoblast cells. As can be seen in Figs 3 and 4, Leu27IGF-II significantly increased tPA, uPA, and MMP-9 expression and significantly reduced TIMP-2 expression in a timedependent manner. It had no effect on MMP-2, TIMP-1, PAI-1, and TGF- $\beta$ protein expression regulation (Figs $3 \mathrm{a}$ and $\mathrm{b}$ and $4 \mathrm{a}$ and $\mathrm{b})$. Moreover, we performed the 
(a)

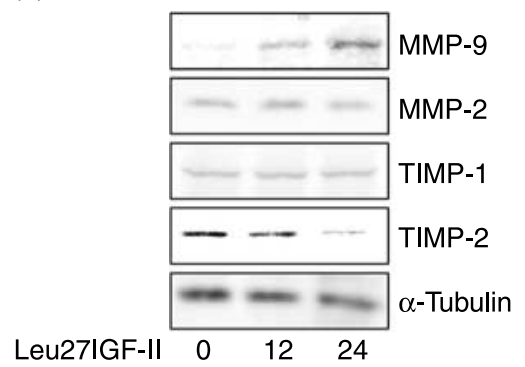

(c)

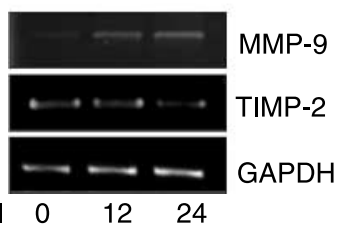

(b)
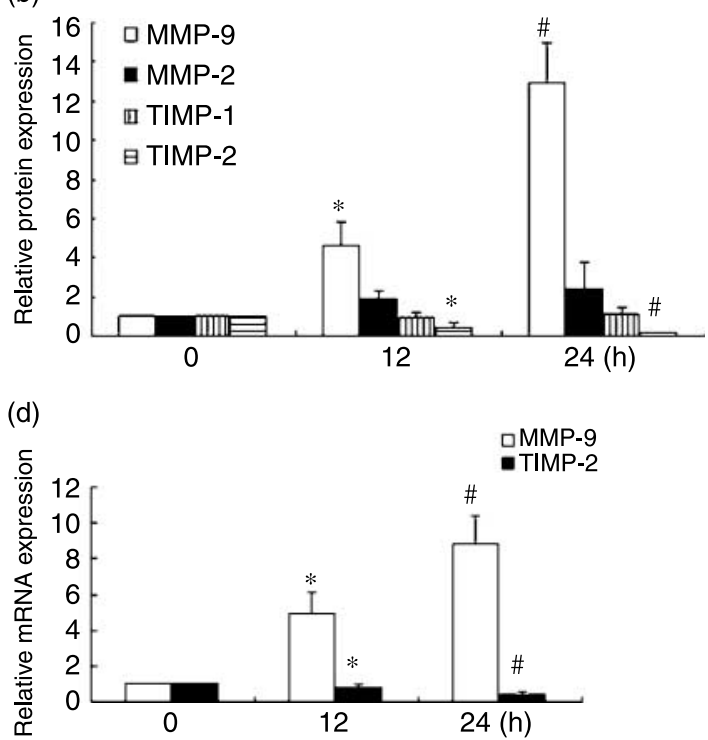

Figure 3 Leu27IGF-II induces the expression of MMP-9/TIMP-2 both at the protein and mRNA level. (a) Western blot analysis of many protein levels, as indicated, in H9c2 cardiomyoblasts treated with Leu27IGF-II $\left(10^{-8} \mathrm{M}\right)$ for 12 and $24 \mathrm{~h}$ showed induction of MMP-9, but suppression of TIMP-2 protein expression in the presence of Leu27IGF-II. $\alpha$-Tubulin served as a loading control. (b) Results are from four independent experiments run in triplicate on cultured cells. Data are quantified by densitometry and presented as means \pm S.E.M. Bars indicate averages. Statistical significance: ${ }^{*} P<0 \cdot 05$; ${ }^{\#} P<0 \cdot 01$, Leu27IGF-II versus untreated controls. (c) RNA transcripts for MMP-9, TIMP-2, and GAPDH (as a internal control) were detected by RT-PCR in H9c2 cardiomyoblasts treated with or without Leu27IGF-II $\left(10^{-8} \mathrm{M}\right)$ for 12 and $24 \mathrm{~h}$. The results showed that induction of the MMP-9 and the suppression of TIMP2 gene expression in the presence of Leu27IGF-II. (d) Data are quantified by densitometry and expressed as fold change of untreated control. Shown are mean results \pm s.E.M. of three independent experiments performed in triplicate. Statistical significance: ${ }^{*} P<0 \cdot 05 ;{ }^{\#} P<0 \cdot 01$, Leu27IGF-II versus untreated control.

RT-PCR assay to detect the mRNA transcripts of tPA, uPA, MMP-9, and TIMP-2 in the H9c2 cardiomyoblast cells treated with Leu27IGF-II. The results in Figs 3c and $\mathrm{d}$ and $4 \mathrm{c}$ and $\mathrm{d}$ show a significant increase in the mRNA level of MMP-9, tPA, and uPA was detected in the cell treated with Leu27IGF-II, whereas the reduction in TIMP-2 mRNA was observed as well. The mRNA levels in the Leu27IGF-II treatment were consistent with protein results (Figs 3 and 4). All the results suggested that Leu27IGF-II may enhance the upregulation of tPA and UPA and induce the MMP-9 activity by disrupting the MMP-9/TIMP-2 balance.

\section{IGF-II activation of MMP-9 activity through IGF2R}

To investigate whether IGF-II-induced MMP-9 activity might be through IGF2R, we further used IGF2R siRNA to disrupt the expression of IGF2R protein in H9c2 cardiomyoblast cells as treated with IGF-II. Western blots showed that there was significantly reduction in the IGF2R protein level in H9c2 cardiomyoblast cells transfected with IGF2R siRNA (Fig. 5a). As shown in Fig. 5b, we found a significantly greater reduction in the
IGF-II-induced activity of MMP-9 in cells transfected with IGF2R siRNA than in cells treated with IGF-II alone. These findings indicated that IGF-II-induced MMP-9 activity was specifically through IGF2R.

\section{Discussion}

In this study, we wanted to find out whether the role of IGF2R in intracellular signaling was involved in maladaptive myocardial remodeling, and if so, whether it occurred through the regulation of ECM degrading enzymes. We found that IGF2R was aberrantly expressed in myocardial infarction scars. Treatment with both IGF-II and Leu27IGF-II, but not IGF-I, in the H9c2 cardiomyoblast cells elevated the MMP-9 activity. Our data further indicated that specific activation of IGF2R by Leu27IGF-II triggered the upregulation of MMP-9, tPA, and UPA and reduced the expression of TIMP2 in H9c2 cardiomyoblast cells as well. Disrupting IGF2R expression inhibited the MMP-9 activity induced by IGF-II. In conclusion, these findings suggest that IGF-II binding to IGF2R may trigger the 
(a)

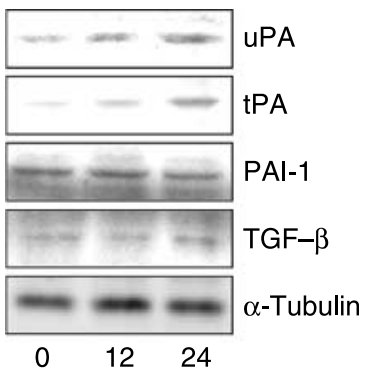

Leu27IGF-II $\quad 0 \quad 12 \quad 24$

(c)

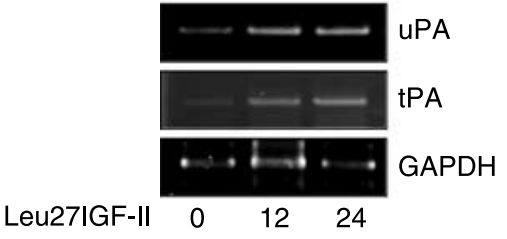

(b)

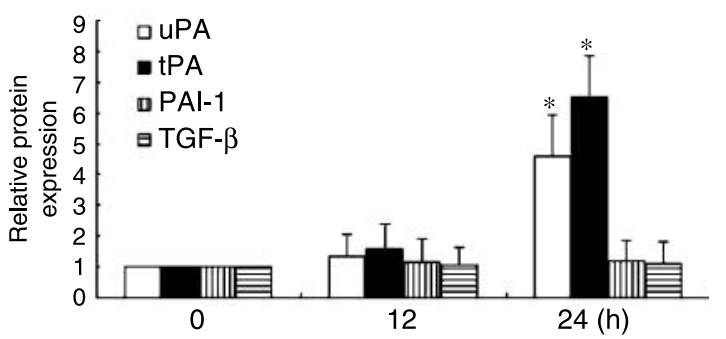

(d)

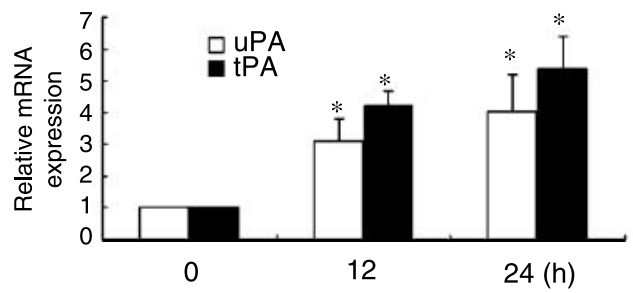

Figure 4 Leu27IGF-II induces the increase of PAs at the level of protein and mRNA. (a) Western blot analysis of many protein levels, as indicated, in H9c2 cardiomyoblast treated with Leu27IGF-II $\left(10^{-8} \mathrm{M}\right)$ for 12 and $24 \mathrm{~h}$ showed upregulation of UPA and tPA protein in the presence of Leu27IGF-II. $\alpha$-Tubulin served as a loading control. (b) Results are from four independent experiments run in triplicate on cultured cells. Data are quantified by densitometry and presented as means \pm S.E.M. Bars indicate averages. Statistical significance: ${ }^{*} P<0 \cdot 05$, Leu27IGF-II versus untreated controls.

(c) Detection of the RNA transcripts of UPA, tPA, and GAPDH (as an internal control) by RT-PCR in the $\mathrm{H} 9 \mathrm{c} 2$ cardiomyoblast cells treated with Leu27IGF-II $\left(10^{-8} \mathrm{M}\right)$. The results showed the induction of the UPA and tPA gene expressions in the presence of Leu27IGF-II. (d) Data are quantified by densitometry and expressed as fold change of untreated control. Shown are mean results \pm S.E.M. of three independent experiments performed in triplicate. Statistical significance: ${ }^{*} P<0.05$; ${ }^{\#} P<0.01$, Leu27IGF-II versus untreated control.

intracellular signaling pathway to increase PA expression (PAs) and then disrupt the balance in MMP-9/TIMP-2 expression level, resulting in increasing MMP-9 activity, contributing to the progression of myocardial remodeling (Fig. 6).

The IGF2R gene is active in the early embryo and expressed widely, but its transcript and protein abundance drop dramatically during the early postnatal period (Schultz et al. 1993). A study of IGF2R-deficient mice reveals that the disruption of IGF2R causes very large and malformed hearts, with marked abnormalities in the ventricular and intraventricular septum (Lau et al. 1994), suggesting the IGF2R play a vital role in normal cardiac morphogenesis and growth in the embryo. However, our study indicated that during cardiac remodeling, IGF2R expression was significantly increased in the myocardial scar tissue when compared with normal heart tissue (Fig. 1), suggesting that overexpressed IGF2R in the adult heart may lead to reprograming of the embryonic gene networks that occurred in the pathological hypertrophy. In a previous study, we found that the upregulation of IGF-II and IGF-II/M6P receptor genes has played a key role in ANGII-induced cell apoptosis and correlated with the promoting of cardiomyocytes apoptosis in hypertensive rat hearts (Lee et al. 2006). It will be of interest in the functional role of IGF2R during cardiac pathologically remodeling in the future.

Previous investigations have reported that IGF2R involvement in ECM remodeling occurs through the proteolytic cleavage of latent TGF- $\beta$ and plasminogen resulting in the activation of TGF- $\beta$ and plasmin (Godar et al. 1999, Ghosh et al. 2003). We found a significant association between IGF2R over expression and myocardial scars (Fig. 1). Furthermore, our results indicated that both IGF-II and Leu27IGF-II, but not IGF-I, induced the increase in MMP-9 activity (Fig. 2). When the IGF2R expression was disrupted by siRNA, the IGF-II-induced MMP-9 activity was rescued (Fig. 5). Their results implied that in addition to trafficking IGF-II to lysosomal degradation, an intracellular signaling cascade could trigger IGF2R binding with IGF-II. Studies have found that through IGF2R signals cross-talk with the small $\mathrm{G}$ protein, they can influence several cellular behaviors, including calcium influx, acetylcholine $(\mathrm{ACh})$ release, and cell migration by activating specific intracellular signaling cascades (Nishimoto et al. 1987, McKinnon et al. 2001, Hawkes et al. 2006). Consistent with our study and those previous investigations, we recommend that IGF2R 

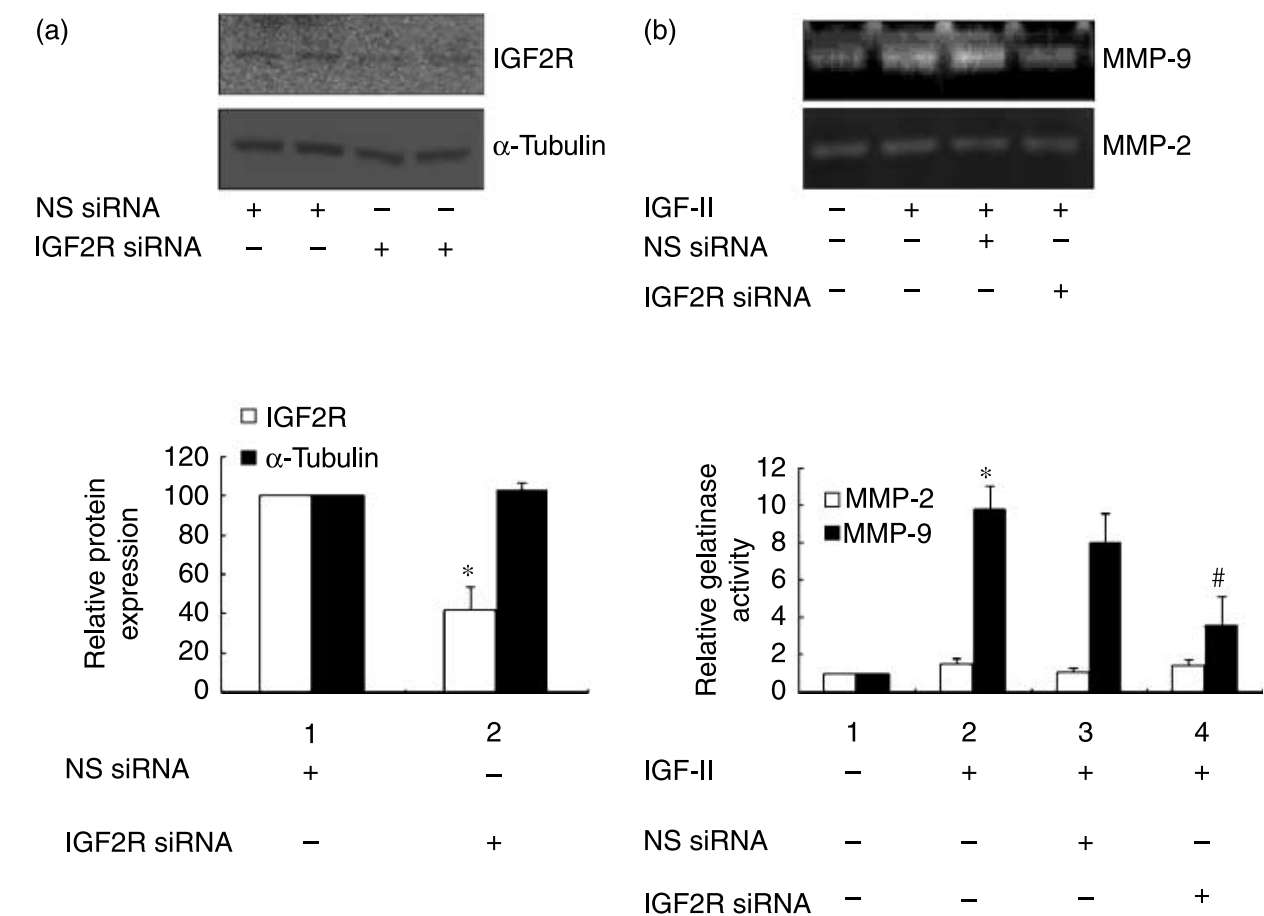

Figure 5 Inhibition of IGF2R expression blocks the IGF-II-induced MMP-9 activity. (a) H9c2 cardiomyoblast cells were transiently transfected with non-specific (NS) siRNA (100 nM) or IGF2Rdirected siRNA ( $100 \mathrm{nM}$ ) for $24 \mathrm{~h}$ to prepare protein lysates and following immunoblotting assay using $\mathrm{G} \alpha \mathrm{q}$ antibody. The results indicated that depletion in IGF2R protein was only determined in IGF2R-directed siRNA transfected cells. Data are quantified by densitometry and expressed as fold change of untreated control. Results are shown as mean \pm S.E.M. of three independent experiments performed in duplicate. Statistical significance: ${ }^{\star} P<0.05$, transfected with IGF2R siRNA versus transfected with NS siRNA controls. (b) The H9c2 cardiomyoblast cells treated with or without IGF-II $\left(10^{-8} \mathrm{M}\right)$ for an additional $24 \mathrm{~h}$ after the transfection with NS siRNA $(100 \mathrm{nM})$ or IGF2R-directed siRNA (100 nM) respectively for $24 \mathrm{~h}$. Gelatin zymography was performed to detect the activity of MMP-2 and MMP-9. The results showed that the MMP-9 activity increased by IGF-II could be rescued by the transfection of IGF2R siRNA. Data were quantified by densitometry and presented as means \pm S.E.M. Bars indicate averages; ${ }^{\#} P<0.05$ values based on comparison with untreated controls; and ${ }^{\star} P<0.05$ values based on comparison with those treated with IGF-II only. $n=3$ independent experiments for each data point.

may play a role in the architectural control of scar tissue formation by the proteolytic cleavage of latent TGF- $\beta$ and plasminogen and also by regulating the intracellular signaling cascades. Further studies are needed to determine whether the activation of specific IGF2R signaling cascades in the heart occurs through the small G-protein-dependent pathway to affect the transcriptional regulation of ECM molecules such as MMPs, TIMPs, and PAs (Deschamps \& Spinale 2006).

The ECM degrading enzyme systems degrade normal collagen structures, change ratios, organization, and cross-links among collagen types, resulting in the scarring process known as cardiac fibrosis ( $\mathrm{Li}$ et al. $2000 b$, Cleutjens \& Creemers 2002). Our results revealed that treatment with Leu27IGF-II, which specifically activated IGF2R signaling, disrupted the normal myocardial MMP-9/TIMP-2 levels (Fig. 3) and increased the expression of PAs (Fig. 4). One study by Ramos-DeSimone et al. (1999) found evidence that the plasminogen system acted as a MMP activity regulator in the heart. That study indicated the active plasmin induced MMP-3 to remove the carboxyl terminus of the proenzyme MMP-9, resulting in its activation (Lijnen et al. 1998, Ramos-DeSimone et al. 1999). The activation of IGF2R signaling might increase the expression of tPA and UPA (Fig. 4), which may in turn promote MMP-9 activity in the H9c2 cardiomyoblast cells (Fig. 2). Moreover, recent findings have shown that inhibition of MMPs or PAs can attenuate the dilatation of the left ventricle in mice with myocardial infarction $(\mathrm{Li}$ et al. 2000b, Peterson et al. 2001, Heymans et al. 2005), suggesting that the inhibition of IGF2R-deriving intracellular signals might be useful in preventing heart failure by regulating ECM remodeling balance.

In conclusion, the results of this study observed the role of IGF2R in intracellular signaling causing myocardial ECM remodeling by altering MMP-9, TIMP-2, and PA expression, and thereby potentially 


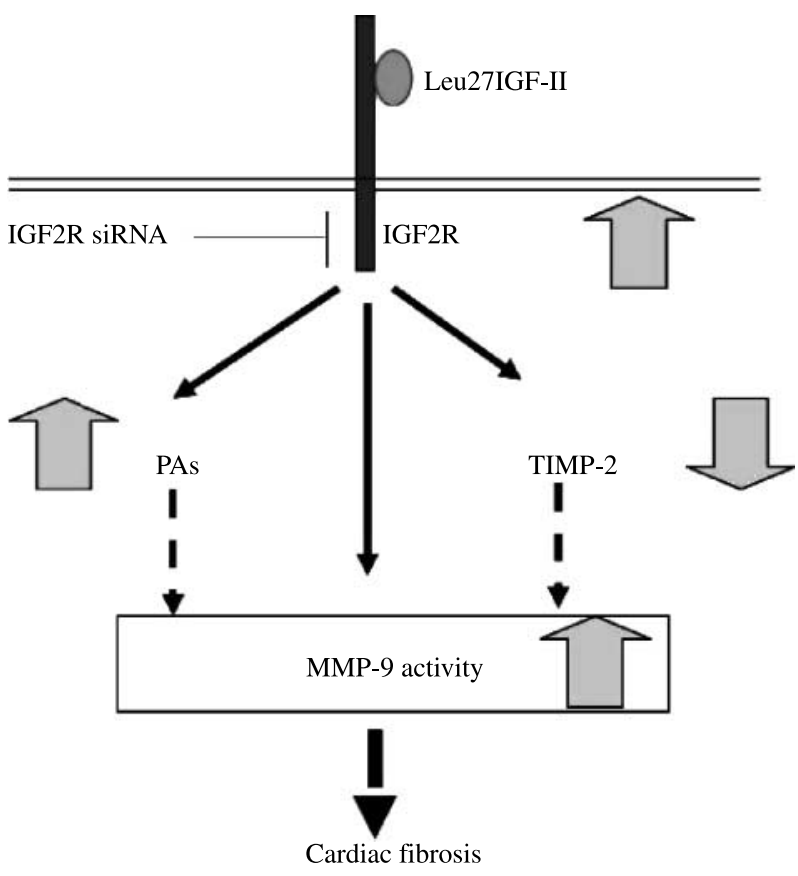

Figure 6 A model for the IGF2R-dependent signaling pathway in cardiac fibrosis. Overexpressed IGF2R significantly associated with the formation of myocardial scars and treatment with IGF-II and Leu27IGF-II, but not IGF-I, can increase in the MMP-9 activity, which implies the IGF2R have intracellular signaling cascades involved in the myocardial remodeling. After binding with Leu27IGF-II, the specific activation of IGF2R signaling affected ECM degrading enzyme systems by disrupting the balance of MMP-9/TIMP-2 expression level and increment of TPA and UPA expression, which in turn may increase MMP-9 activity. Therefore, disruption of IGF2R expression by siRNA significantly inhibited the IGF-II-induced MMP-9 activity, suggesting IGF2R signaling may play a critical role in the progression of myocardial fibrosis. Solid arrows denote pathways observed in this study. Dotted lines denote possible pathways that were not examined.

inducing MMP-9 activity. These new insights may be used into preventing chronic heart disease associated with fibrosis.

\section{Acknowledgements}

The authors declare that there is no conflict of interest that would prejudice the impartiality of this scientific work.

\section{References}

Berk BC, Fujiwara K \& Lehoux S 2007 ECM remodeling in hypertensive heart disease. Journal of Clinical Investigation 117 $568-575$.

Beukers MW, Oh Y, Zhang H, Ling N \& Rosenfeld RG 1991 [Leu27] insulin-like growth factor II is highly selective for the type-II IGF receptor in binding, cross-linking and thymidine incorporation experiments. Endocrinology 128 1201-1203.
Chen Z, Ge Y \& Kang JX 2004 Down-regulation of the M6P/IGF-II receptor increases cell proliferation and reduces apoptosis in neonatal rat cardiac myocytes. BMC Cell Biology 515.

Cleutjens JP \& Creemers EE 2002 Integration of concepts: cardiac extracellular matrix remodeling after myocardial infarction. Journal of Cardiac Failure 8 S344-S348.

Deschamps AM \& Spinale FG 2006 Pathways of matrix metalloproteinase induction in heart failure: bioactive molecules and transcriptional regulation. Cardiovascular Research 69 666-676.

Ducharme A, Frantz S, Aikawa M, Rabkin E, Lindsey M, Rohde LE, Schoen FJ, Kelly RA, Werb Z, Libby P et al. 2000 Targeted deletion of matrix metalloproteinase-9 attenuates left ventricular enlargement and collagen accumulation after experimental myocardial infarction. Journal of Clinical Investigation 106 55-62.

Fay WP 2004 Plasminogen activator inhibitor 1, fibrin, and the vascular response to injury. Trends in Cardiovascular Medicine $\mathbf{1 4}$ 196-202.

Gaertner R, Jacob MP, Prunier F, Angles-Cano E, Mercadier IJ \& Michel JB 2005 The plasminogen-MMP system is more activated in the scar than in viable myocardium 3 months post-MI in the rat. Journal of Molecular and Cellular Cardiology 38 193-204.

Gallagher GL, Jackson CJ \& Hunyor SN 2007 Myocardial extra cellular matrix remodeling in ischemic heart failure. Frontiers in Bioscience 12 $1410-1419$

Ghosh P, Dahms NM \& Kornfeld S 2003 Mannose 6-phosphate receptors: new twists in the tale. Nature Reviews. Molecular Cell Biology 4 202-212.

Godar S, Horejsi V, Weidle UH, Binder BR, Hansmann C \& Stockinger H 1999 M6P/IGFII-receptor complexes urokinase receptor and plasminogen for activation of transforming growth factor-betal. European Journal of Immunology 29 1004-1013.

Hawkes C, Jhamandas JH, Harris KH, Fu W, MacDonald RG \& Kar S 2006 Single transmembrane domain insulin-like growth factorII/mannose-6-phosphate receptor regulates central cholinergic function by activating a G-protein-sensitive, protein kinase C-dependent pathway. Journal of Neuroscience 26 585-596.

Heymans S, Luttun A, Nuyens D, Theilmeier G, Creemers E, Moons L, Dyspersin GD, Cleutjens JP, Shipley M, Angellilo A et al. 1999 Inhibition of plasminogen activators or matrix metalloproteinases prevents cardiac rupture but impairs therapeutic angiogenesis and causes cardiac failure. Nature Medicine 5 1135-1142.

Heymans S, Lupu F, Terclavers S, Vanwetswinkel B, Herbert JM, Baker A, Collen D, Carmeliet P \& Moons L 2005 Loss or inhibition of UPA or MMP-9 attenuates LV remodeling and dysfunction after acute pressure overload in mice. American Journal of Pathology 166 $15-25$.

Jones JI \& Clemmons DR 1995 Insulin-like growth factors and their binding proteins: biological actions. Endocrine Reviews 16 3-34.

Lau MM, Stewart CE, Liu Z, Bhatt H, Rotwein P \& Stewart CL 1994 Loss of the imprinted IGF2/cation-independent mannose 6 -phosphate receptor results in fetal overgrowth and perinatal lethality. Genes and Development 8 2953-2963.

Leask A \& Abraham DJ 2004 TGF-beta signaling and the fibrotic response. FASEB Journal 18 816-827.

Lee SD, Chu CH, Huang EJ, Lu MC, Liu JY, Liu CJ, Hsu HH, Lin JA, Kuo WW \& Huang CY 2006 Roles of insulin-like growth factor II in cardiomyoblast apoptosis and in hypertensive rat heart with abdominal aorta ligation. American Journal of Physiology. Endocrinology and Metabolism 291 E306-E314.

Li YY, McTiernan CF \& Feldman AM 2000 $a$ Interplay of matrix metalloproteinases, tissue inhibitors of metalloproteinases and their regulators in cardiac matrix remodeling. Cardiovascular Research 46 214-224.

Li H, Simon H, Bocan TM \& Peterson JT $2000 b$ MMP/TIMP expression in spontaneously hypertensive heart failure rats: the effect of ACE- and MMP-inhibition. Cardiovascular Research 46 298-306. 
Lijnen HR, Silence J, Lemmens G, Frederix L \& Collen D 1998 Regulation of gelatinase activity in mice with targeted inactivation of components of the plasminogen/plasmin system. Thrombosis and Haemostasis 79 1171-1176.

McKinnon T, Chakraborty C, Gleeson LM, Chidiac P \& Lala PK 2001 Stimulation of human extra villous trophoblast migration by IGF-II is mediated by IGF type 2 receptor involving inhibitory $\mathrm{G}$ protein (s) and phosphorylation of MAPK. Journal of Clinical Endocrinology and Metabolism 86 3665-3674.

Nishimoto I, Hata Y, Ogata E \& Kojima I 1987 Insulin-like growth factor II stimulates calcium influx in competent BALB/c 3T3 cells primed with epidermal growth factor. Characteristics of calcium influx and involvement of GTP-binding protein. Journal of Biological Chemistry 262 12120-12126.

Odekon LE, Blasi F \& Rifkin DB 1994 Requirement for receptor-bound urokinase in plasmin-dependent cellular conversion of latent TGF- $\beta$ to TGF- $\beta$. Journal of Cellular Physiology 158 398-407.

Peterson JT, Hallak H, Johnson L, Li H, O'Brien PM, Sliskovic DR, Bocan TM, Coker ML, Etoh T \& Spinale FG 2001 Matrix metalloproteinase inhibition attenuates left ventricular remodeling and dysfunction in a rat model of progressive heart failure. Circulation 103 2303-2309.
Ramos-DeSimone N, Hahn-Dantona E, Sipley J, Nagase H, French DL \& Quigley JP 1999 Activation of matrix metalloproteinase-9 (MMP-9) via a converging plasmin/stromelysin-1 cascade enhances tumor cell invasion. Journal of Biological Chemistry 274 13066-13076.

Schultz GA, Hahnel A, Arcellana-Panlilio M, Wang L, Goubau S, Watson A \& Harvey M 1993 Expression of IGF ligand and receptor genes during preimplantation mammalian development. Molecular Reproduction and Development 35 414-420.

Spinale FG 2002 Matrix metalloproteinases: regulation and dysregulation in the failing heart. Circulation Research 90 520-530.

Spinale FG, Coker ML, Heung LJ, Bond BR, Gunasinghe HR, Etoh T, Goldberg AT, Zellner JL \& Crumbley AJ 2000 A matrix metalloproteinase induction/activation system exists in the human left ventricular myocardium and is upregulated in heart failure. Circulation 102 1944-1949.

Received in final form 15 May 2008 Accepted 20 May 2008

Made available online as an Accepted Preprint 20 May 2008 